\title{
In vivo functional human imaging using photoacoustic microscopy: response to ischemic and thermal stimuli
}

Christopher Favazza, Konstantin Maslov, Lynn Cornelius, Lihong V. Wang

Christopher Favazza, Konstantin Maslov, Lynn Cornelius, Lihong V. Wang, "In vivo functional human imaging using photoacoustic microscopy: response to ischemic and thermal stimuli," Proc. SPIE 7564, Photons Plus Ultrasound: Imaging and Sensing 2010, $75640 Z$ (23 February 2010); doi: $10.1117 / 12.841975$ 


\title{
In vivo functional human imaging using photoacoustic microscopy: response to ischemic and thermal stimuli
}

\author{
Christopher Favazza ${ }^{\mathrm{a}}$, Konstantin Maslov ${ }^{\mathrm{a}}$, Lynn Cornelius ${ }^{\mathrm{b}}$, Lihong V. Wang ${ }^{\mathrm{a},{ }^{*}}$ \\ ${ }^{a}$ Optical Imaging Laboratory, Department of Biomedical Engineering, \\ Washington University in St. Louis, MO USA 63130; \\ ${ }^{b}$ Division of Dermatology, Washington University School of Medicine, St. Louis, MO USA 63110
}

\begin{abstract}
We report results of two in vivo functional human imaging experiments using photoacoustic microscopy. In Experiment 1, the hemodynamic response to an ischemic event was measured. The palm of a volunteer was imaged and a single cross-section was monitored while periodic arterial occlusions were administered using a blood pressure cuff wrapped around the upper arm and inflated to $\sim 280 \mathrm{mmHg}$. Significant relative decreases in oxygen saturation $\left(\mathrm{sO}_{2}\right)$ and total hemoglobin $(\mathrm{HbT})$ were observed during periods of ischemia. Upon release of the occlusion, significant relative increases in $\mathrm{sO}_{2}$ and $\mathrm{HbT}$ due to post-occlusive reactive hyperemia were recorded. Experiment 2 explored the vascular response to a local, external thermal stimulus. Thermal hyperemia is a common physiological phenomenon and thermoregulation function in which blood flow to the skin is increased to more efficiently exchange heat with the ambient environment. The forearm of a volunteer was imaged and a single cross-section was monitored while the imaged surface was exposed to an elevated temperature of $\sim 46^{\circ} \mathrm{C}$. Due to thermal hyperemia, relative increases in $\mathrm{sO}_{2}$ and $\mathrm{HbT}$ were measured as the temperature of the surface was raised. These results may contribute as clinically relevant measures of vascular functioning for detection and assessment of vascular related diseases.
\end{abstract}

Keywords: photoacoustic microscopy, reactive hyperemia, microcirculation

\section{INTRODUCTION}

Microvascular networks are becoming increasingly important for signaling health problems in an individual. The microvasculature provides a window into systemic cardiovascular complications. And microvascular functioning could be used as a mechanism whereby cardiovascular related diseases could be diagnosed or assessed (1-5). A simple and repeatable method of evaluating vascular functioning is to perturb circulation and measure the response. There are two common methods of probing the microvascular system. One method involves temporarily occluding blood flow and measuring the post-occlusive reactive hyperemia (PORH) response $(6,7)$. In short, PORH is the transient increase of local perfusion that results from an ischemic event, specifically from the build-up of metabolic waste and low oxygenation $(3,4,8)$. In a second measurement technique, a local region of the skin is heated and the ensuing local thermal hyperemia is evaluated $(1,9)$. Thermal hyperemia is the thermoregulative response to elevated temperatures in which there is an increase in perfusion to superficial vascular networks and to lower body temperature. In daily life, this process is stimulated by an increase in the core body temperature or ambient temperature, and is usually accompanied by sweating. As more blood flows to the surface it is more efficiently cooled by evaporating sweat, thereby exchanging heat from the core with the ambient environment. This phenomenon can also be triggered through local warming of the skin $(3,9)$. By heating the skin to temperature around $42^{\circ} \mathrm{C}$, perfusion can increase several fold (1).

Generally, non-invasive measurements of microcirculation are performed using near-infrared spectroscopy, laser Doppler, or plethysmography $(1,4,8,10,11)$. Often, these techniques are limited to single parameter measurements, $\mathrm{sO}_{2}$, perfusion/changes in blood volume, or blood flow. Likewise, these methods usually do not resolve the vessels from which the aforementioned parameters are measured. However, with photoacoustic microscopy it is possible to simultaneously measure multiple parameters, while providing a high resolution image of the measured microvasculature.

* author for correspondence: lhwang@seas.wustl.edu

Photons Plus Ultrasound: Imaging and Sensing 2010, edited by Alexander A. Oraevsky, Lihong V. Wang, Proc. of SPIE Vol. 7564, 75640Z · C 2010 SPIE · CCC code: 1605-7422/10/\$18 · doi: 10.1117/12.841975 
Numerous reports have detailed the capabilities of functional photoacoustic microscopy, particularly for hemodynamic measurements in animal models (12-14). For instance, vascular responses to changes in the oxygen concentration supplied to mice have been measured in superficial microvascular networks in the mouse ear and larger vessels in the brain $(12,13)$. Both reports clearly indicate changes in the blood volume and oxygen saturation $\left(\mathrm{sO}_{2}\right)$ that correspond with changes in the oxygen concentration of the inhalation gas. To date, similar studies involving perturbing microcirculation have been limited to animals, and have not been applied to humans. In this paper, we present results from two sets of functional photoacoustic imaging experiments applied to human cutaneous microvascular networks: post-occlusive reactive hyperemia and thermal hyperemia experiments.

\section{METHODS AND MATERIALS}

\subsection{Experiment 1: Post-occlusive reactive hyperemia}

In this experiment, a custom built photoacoustic macroscope was employed to image the microvascular structure and functioning in five different volunteers. An Edgewave Nd:YLF laser supplied the optical energy to probe the sample, and pumped a Sirah dye laser. We used Rhodamine 6G in the dye laser to generate wavelengths between $560 \mathrm{~nm}$ and $575 \mathrm{~nm}$. The pulse width of the beam was less than $5 \mathrm{~ns}$ and the pulse repetition rate, which depends on the motorized scanning parameters, was approximately $1 \mathrm{kHz}$. The laser beam was then coupled into a fiber optic cable, and was routed to the PAM system. The light was weakly focused into sample using dark-field illumination. More details of this system can be found elsewhere $(15,16)$. A focused, broadband transducer with a $20 \mathrm{MHz}$ central frequency was confocally aligned with the weak focus of the laser light. With this set-up and transducer, it is possible to achieve $70 \mu \mathrm{m}$ lateral resolution and $54 \mu \mathrm{m}$ axial resolution (17).

In this set of experiments, cutaneous microvascular networks in the palm of the skin were investigated. The experiment consisted of a three-dimensional imaging portion and a functional imaging portion. First, the palm of a volunteer was placed in acoustic contact with a water tank that housed the ultrasonic transducer. An area of $8 \times 4 \mathrm{~mm}$ on the lower palm of the volunteer was scanned and two three-dimensional images were acquired, one using $570 \mathrm{~nm}$ light and the other using $561 \mathrm{~nm}$ light. The images were acquired within approximately fifteen minutes. Following the 3-D image acquisitions, a specific cross-section or b-scan was selected from the dataset. This cross-section was repeatedly scanned over the course of fifteen minutes during which two ischemic events were periodically induced. The two arterial occlusions were created by inflating an arm cuff to a standard pressure of $\sim 280 \mathrm{mmHg}$ for each volunteer. The occlusions were initiated after 3 and 9 minutes into the experiment. The b-scan images were generated with laser wavelengths of $561 \mathrm{~nm}$ and $570 \mathrm{~nm}$. The time between the images at both wavelengths was approximately one second, due to the mechanical time required to tune the wavelength. The dual wavelength images were initiated every fifteen seconds.

\subsection{Experiment 2: Thermal hyperemia}

For this set of experiments, the same dark-field imaging system was employed. In these experiments, the transducer from Experiment 1 was replaced by a transducer with a $50 \mathrm{MHz}$ central frequency. This system configuration and transducer yield $45 \mu \mathrm{m}$ lateral resolution and $15 \mu \mathrm{m}$ axial resolution.

The ventral side of the forearm of a volunteer was placed in acoustic contact with the water tank and indirectly the ultrasound transducer. A single cross-section was scanned for 20 minutes using two different laser wavelengths, $561 \mathrm{~nm}$ and $570 \mathrm{~nm}$. Baseline measurements were recorded for the first 1.5 minutes of the experiment, after which the skin of the volunteer was warmed by heating the water in the water tank to approximately $46^{\circ} \mathrm{C}$. Again, wavelength tuning resulted in approximately one second between scans at both wavelengths. In this experiment, dual wavelength image acquisitions were initiated every 10 seconds. To induce local thermal hyperemia, a thin, coiled steel tube was used to circulate hot water, heat the water in the water tank, and indirectly warm the skin of the volunteer. Water in the reservoir of the circulator was pre-heated prior to the experiment for more efficient and repeatable heating timescales.

Additionally, an automated, dynamic z-axis repositioning system was incorporated into the imaging protocol. This additional function corrected for changes in the focal length of the focused transducer as the speed of sound of the water in the tank increase with the rise in temperature. A reference point of ink was marked on the forearm of the volunteer, which was located at the edge of the lateral dimension of the b-scan image. The depth position of this point was calculated during scanning and used to adjust the depth of the transducer accordingly. 


\section{RESULTS AND DISCUSSION}

\subsection{Post-occlusive reactive hyperemia}

During these experiments, several changes in the microcirculation were measured. There were clear differences between the photoacoustic signals before, during, and after the occlusions. Likewise, the ratio between the photoacoustic signals drastically changed during and after the occlusions. Figures $1(\mathrm{a}-\mathrm{b})$ show the images acquired using $570 \mathrm{~nm}$ light including a maximum amplitude projection of a 3-D image and the selected b-scan for monitoring during the functional imaging portion of the experiment. Figures $1(\mathrm{c}-\mathrm{d})$ show the pseudo m-mode images collected using both $561 \mathrm{~nm}$ and 570 $\mathrm{nm}$ light. In each image (Fig. 1(c-d)), the maximum amplitude of each b-scan is projected on the $\mathrm{x}$-axis for each timepoint measurement during the 15 minute monitoring period. It is clearly evident that there are drastic changes in the measured photoacoustic signal during and after the occlusions.
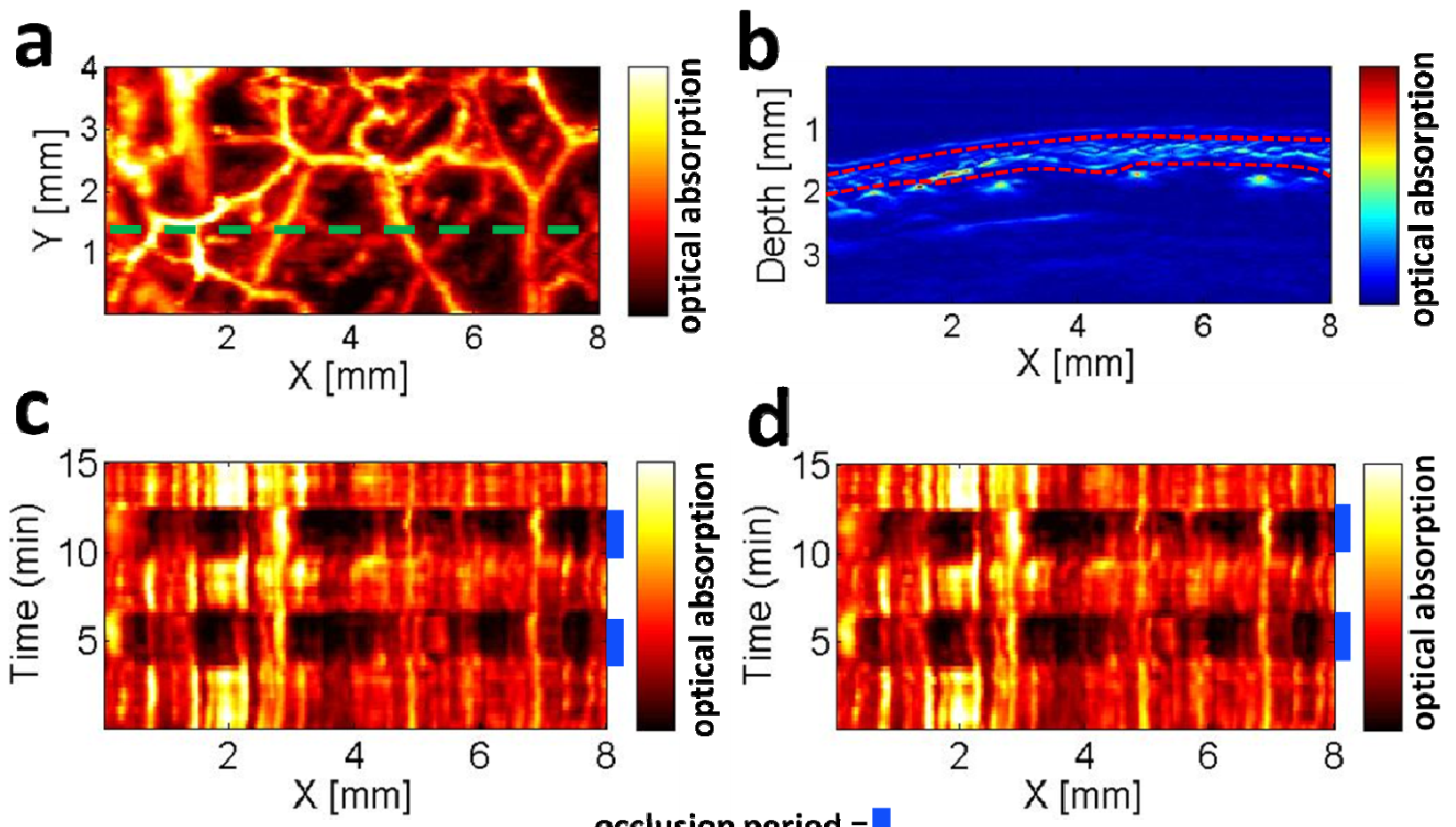

Figure 1. Resulting images from of the post-occlusive reactive hyperemia experiment. (a) Maximum amplitude projection of the 3-D photoacoustic image acquired with $570 \mathrm{~nm}$ laser light. The dashed green line indicates the cross-section displayed in (b). (b) B-scan image of the selected cross-section that was monitored during the functional imaging portion of the experiment. The red dashed lines mark the capillary bed between them, from which data was collected and analyzed. (c) and (d) The maximum amplitude of each b-scan, time-point measurement projected onto the $\mathrm{x}$-axis for data obtained at $570 \mathrm{~nm}$ and $561 \mathrm{~nm}$, respectively. The blue bars on the sides of the images indicate the occlusion periods.

Measurements were obtained from signals generated within the capillary bed in the b-scans. This region is in between the dashed red lines in Figure 1b. The capillary bed was selected for analysis in order to evaluate relative changes in the $\mathrm{sO}_{2}$; whereas other vessels, such as arterials or venules, are not expected to experience a large change in the relative $\mathrm{sO}_{2}$ since oxygen is primarily exchanged through capillary vessels. The plot in Figure 2(a) shows the effects of the periodic occlusions on $\mathrm{sO}_{2}$. Specifically, the ratio of the photoacoustic signals obtained with a deoxyhemoglobin-dominant wavelength $(561 \mathrm{~nm})$ and an isosbestic wavelength $(570 \mathrm{~nm})$ is plotted in time and normalized to the initial baseline measurements. At the isosbesitc wavelength, the absorption coefficient of oxyhemoglobin equals that of deoxyhemoglobin, thus the ratio of the photoacoustic signals acquired at these wavelengths indicate changes in the 
deoxyhemoglobin concentration of the blood, and indirectly the $\mathrm{sO}_{2}$. During the occlusion, the relative deoxyhemoglobin concentration increases, while immediately following the release of the occlusion it decreases. An average of $\sim 19 \%$ relative increase was measured during the first occlusion and an average of $\sim 18 \%$ relative increase was found during the second occlusion. Conversely, an average relative decrease in deoxyhemoglobin of $\sim 11 \%$ was measured after the release of the first and second occlusion. These results are consistent with physiological expectations, in which the $\mathrm{sO}_{2}$ decreases as the blood flow is occluded, and increase during the hyperemic state following the release of the occlusion.
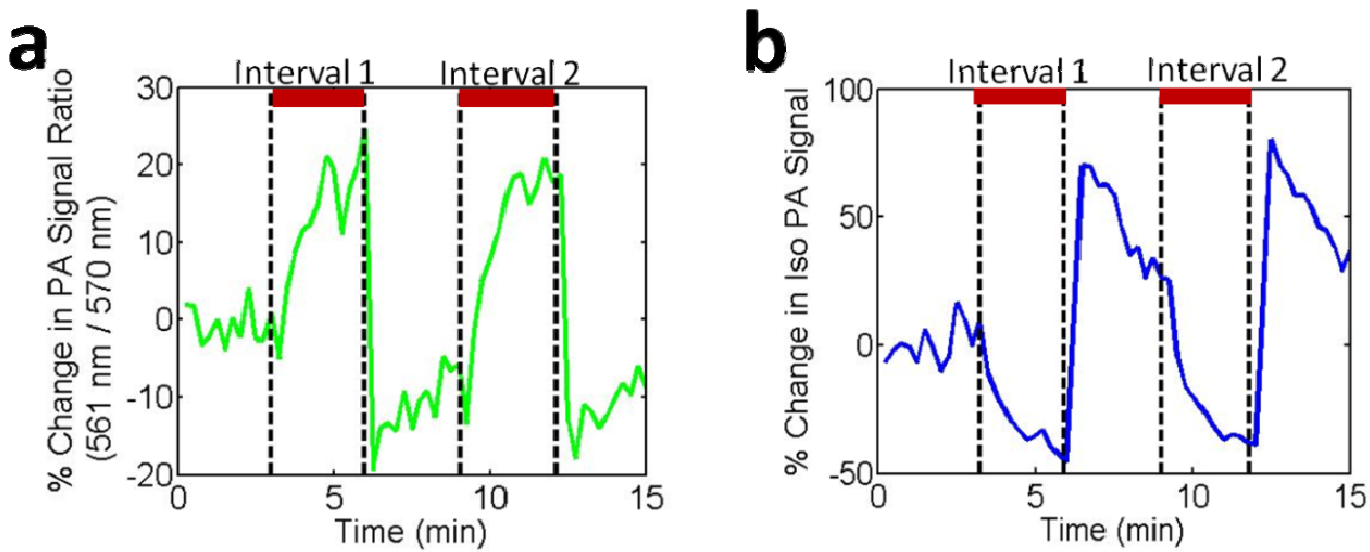

Figure 2. Data analysis from the post-occlusive reactive hyperemia experiment: (a) A plot of the ratio of the photoacoustic signals acquired at $561 \mathrm{~nm}$ and $570 \mathrm{~nm}$ for each time-point and normalized to the initial baseline measurements. This plot shows the relative change in the deoxyhemoglobin concentration as related to the applied occlusions. (b) A plot of the isosbestic photoacoustic signal acquired at $570 \mathrm{~nm}$ and normalized to the initial baseline measurements. This plot shows relative changes in the total amount of hemoglobin or blood volume. The red bars in each plot mark the occlusion periods.

Release of the occlusion resulted in PORH. Analysis of this process measured in the capillary bed of the volunteer is shown in Figure 2(b). In the graph, changes in the photoacoustic signal acquired at an isosbestic wavelength $(570 \mathrm{~nm})$ are plotted in time and normalized to the baseline measures. These relative changes in the photoacoustic signal indicate fluctuations in the total amount of hemoglobin or blood volume. Upon release of the occlusion the photoacoustic signal increased. A maximum relative increase in signal of $\sim 70 \%$ was measured after the first occlusion and $\sim 80 \%$ after the second occlusion. These results are qualitatively consistent with known physiological phenomenon where perfusion greatly increases after an ischemic event.

\subsection{Local thermal hyperemia}

Figure 3 shows the results from the thermal hyperemia experiment. Two b-scans, one acquired at the beginning of the experiment and another 7.5 minutes into the experiment, are displayed in Figures 3(a-b). Both b-scans were acquired with $570 \mathrm{~nm}$ illumination. The temperature of the water in the water tank was approximately $\sim 24^{\circ} \mathrm{C}$ in the beginning of the experiment and reached $\sim 46^{\circ} \mathrm{C}$ by $\sim 7$ minutes into the experiment. An increase in the photoacoustic signal (and area of signal) can be seen in the transition from Figure 3(a) to Figure 3(b), which marks the increase in perfusion. Similarly, the images in Figures 3(c-d), in which the maximum amplitudes of each b-scan are projected onto the x-axis for each time-point measurement, show an increase in photoacoustic signal. 

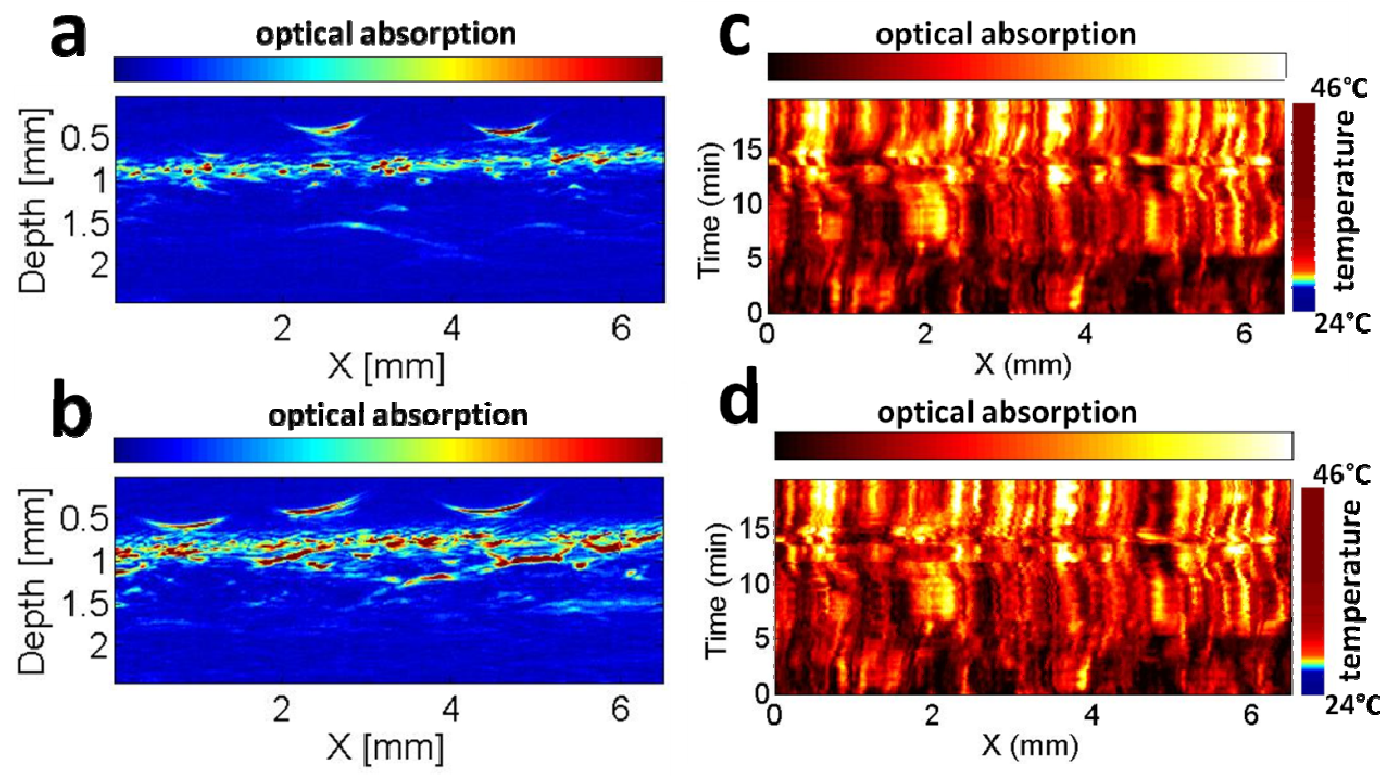

Figure 3. Resulting images from the thermal hyperemia experiment. (a) B-scan of the monitored cross-section at the beginning of the experiment with a water tank temperature of approximately $24^{\circ} \mathrm{C}$. (b) B-scan image of the monitored cross-section 7.5 minutes into the experiment with a water tank temperature of approximately $46^{\circ} \mathrm{C}$. (c) and (d) The maximum amplitude of the b-scans at each time-point measurement projected onto the $\mathrm{x}$-axis for data collected at $570 \mathrm{~nm}$ and $561 \mathrm{~nm}$, respectively. The colorbar on the left indicates the temperature profile of the water tank in time.

Figure 4 shows the analysis of the photoacoustic data collected from the capillary beds of the volunteer during local thermal hyperemia. In Figure 4(a), relative changes in the deoxyhemoglobin concentration are shown. Also plotted in the graph is the thermal profile of the water in the water tank during the experiment. The data show that there is a relative decrease in deoxyhemoglobin concentration as the skin is warmed. In Figure 4(b), relative changes in the isosbestic photoacoustic signal are plotted along with the corresponding temperature profiles. As seen in the plot, the photoacoustic signal drastically increases after the skin surface is exposed to an elevated temperature. This relative change in the photoacoustic signal marks an increase in perfusion of capillaries, which is consistent with the expected response to local thermal stimulus.
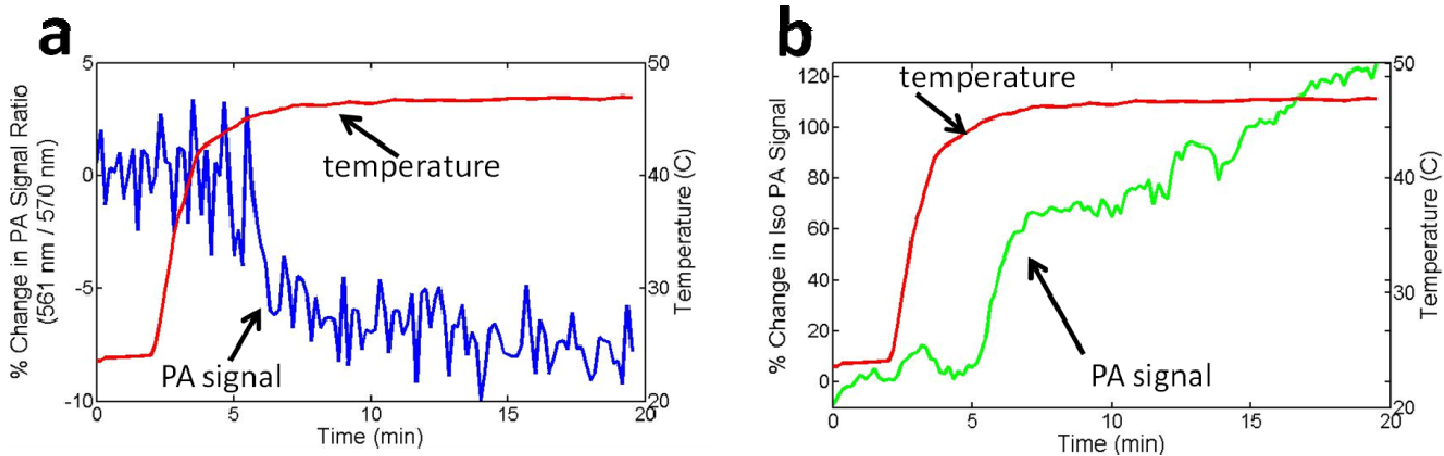

Figure 4. Data analysis from the thermal hyperemia experiment. (a) A plot of the ratio of photoacoustic signals obtained at $561 \mathrm{~nm}$ and $570 \mathrm{~nm}$ and normalized to the initial baseline measurements. Photoacoustic data (green) is plotted along with the thermal profile (red) with the temperature values displayed on the right. (b) A plot of the isosbestic photoacoustic signal, acquired at $570 \mathrm{~nm}$, and normalized to the initial baseline measurements. Photoacoustic data (blue) is plotted along with the thermal profile (red) with the temperature values displayed on the right. 


\section{CONCLUSIONS}

In the PORH experiment relative increases in deoxyhemoglobin concentration and decreases in perfusion were measured during the periods of ischemia, and relative decreases in deoxyhemoglobin concentration and increases in perfusion were observed following the occlusions. In the thermal hyperemia experiment, a relative decrease in deoxyhemoglobin concentration and an increase in perfusion were measured. The results from both experiments demonstrate the potential of photoacoustic microscopy as a tool to measure microvascular functioning.

\section{ACKNOWLEDGEMENTS}

This research was supported by National Institutes of Health grants R01 EB000712, 5 T32 AR07284, R01 EB008085, R01 CA113453901, and U54 CA136398. LW acknowledges financial interest in Endra Inc., which, however, did not support this research.

\section{REFERENCES}

[1] N. Charkoudian, "Skin blood flow in adult human thermoregulation: how it works, when it does not, and why," Mayo Clin Proc. 78, pp.603-612, (2003)

[2] X. Cheng, J. M. Mao, X. Xu, M. Elmandjra, R. Bush, L. Christenson, B. O'Keefe and J. Bry, "Post-occlusive reactive hyperemia in patients with peripheral vascular disease," Clin Hemorheol Microcirc. 31, pp.11-21, (2004)

[3] J. L. Cracowski, C. T. Minson, M. Salvat-Melis and J. R. Halliwill, "Methodological issues in the assessment of skin microvascular endothelial function in humans," Trends Pharmacol Sci. 27, pp.503-508, (2006)

[4] B. Fagrell and M. Intaglietta, "Microcirculation: its significance in clinical and molecular medicine," $J$ Intern Med. 241, pp.349-362, (1997)

[5] A. Kruger, J. Stewart, R. Sahityani, E. O'Riordan, C. Thompson, S. Adler, R. Garrick, P. Vallance and M. S. Goligorsky, "Laser Doppler flowmetry detection of endothelial dysfunction in end-stage renal disease patients: correlation with cardiovascular risk," Kidney Int. 70, pp.157-164, (2006)

[6] T. Hamaoka, T. Katsumura, N. Murase, S. Nishio, T. Osada, T. Sako, H. Higuchi, Y. Kurosawa, T. Shimomitsu, M. Miwa and B. Chance, "Quantification of ischemic muscle deoxygenation by near infrared time-resolved spectroscopy," J Biomed Opt. 5, pp.102-105, (2000)

[7] N. B. Hampson and C. A. Piantadosi, "Near infrared monitoring of human skeletal muscle oxygenation during forearm ischemia," J Appl Physiol. 64, pp.2449-2457, (1988)

[8] F. F. de Mul, F. Morales, A. J. Smit and R. Graaff, "A model for post-occlusive reactive hyperemia as measured with laser-Doppler perfusion monitoring," IEEE Trans Biomed Eng. 52, pp.184-190, (2005)

[9] B. J. Wong, S. J. Williams and C. T. Minson, "Minimal role for H1 and H2 histamine receptors in cutaneous thermal hyperemia to local heating in humans," J Appl Physiol. 100, pp.535-540, (2006)

[10] G. Addor, A. Delachaux, B. Dischl, D. Hayoz, L. Liaudet, B. Waeber and F. Feihl, "A comparative study of reactive hyperemia in human forearm skin and muscle," Physiol Res. 57, pp.685-692, (2008)

[11] B. Chance, S. Nioka, J. Kent, K. McCully, M. Fountain, R. Greenfeld and G. Holtom, "Time-resolved spectroscopy of hemoglobin and myoglobin in resting and ischemic muscle," Anal Biochem. 174, pp.698-707, (1988)

[12] S. Hu, K. Maslov and L. V. Wang, "Noninvasive label-free imaging of microhemodynamics by opticalresolution photoacoustic microscopy," Opt Express. 17, pp.7688-7693, (2009)

[13] E. W. Stein, K. Maslov and L. V. Wang, "Noninvasive, in vivo imaging of blood-oxygenation dynamics within the mouse brain using photoacoustic microscopy," J Biomed Opt. 14, pp.020502, (2009)

[14] H. F. Zhang, K. Maslov, G. Stoica and L. V. Wang, "Functional photoacoustic microscopy for high-resolution and noninvasive in vivo imaging," Nat Biotechnol. 24, pp.848-851, (2006)

[15] K. Maslov, G. Stoica and L. V. Wang, "In vivo dark-field reflection-mode photoacoustic microscopy," Opt Lett. 30, pp.625-627, (2005)

[16] H. F. Zhang, K. Maslov and L. V. Wang, "In vivo imaging of subcutaneous structures using functional photoacoustic microscopy," Nat Protoc. 2, pp.797-804, (2007)

[17] E. W. Stein, K. Maslov and L. V. Wang, "Noninvasive, in vivo imaging of the mouse brain using photoacoustic microscopy," J Appl Phys. 105, pp.102027, (2009) 